\title{
Structural and Chemical Characterisation of the Biomineralized Teeth in Marine Molluscs Using Focused Ion Beam (FIB) Processing and TEM
}

\author{
M. Saunders, ${ }^{*}$ C. Kong ${ }^{* *}$, J. A. Shaw, ${ }^{*}$ D. J. Macey, ${ }^{* * *}$ P. L. Clode* \\ * Centre for Microscopy, Characterisation and Analysis, The University of Western Australia, \\ Crawley, Perth, WA 6009, Australia \\ ** Electron Microscopy Unit, University of New South Wales, Kingsford, NSW 2052, Australia \\ *** School of Biological Sciences \& Biotechnology, Murdoch University, Murdoch, WA 6150, \\ Australia
}

Understanding biomineralization processes could provide a means to produce novel biomimetic materials with potential applications in a diverse range of fields from medicine to materials engineering. The teeth of chitons (marine molluscs) represent an excellent example of a composite biomineralized structure, comprising variable layers of iron oxide, iron oxyhydroxide and apatite. While the early stages of the biomineralization process can be well characterised by a variety of microscopy and microanalytical techniques (see, for example, Shaw, et al. elsewhere in these proceedings and [1]), the hard, fully mineralized teeth are a more difficult proposition.

Studies of mineralized teeth using techniques such as X-ray diffraction, Raman spectroscopy and scanning electron microscopy (SEM) have provided tantalising glimpses of the underlying microstructure [2], but have lacked the resolution to provide vital information on the fine scale structure and chemistry, particularly at interfaces. Transmission electron microscopy (TEM) has the potential to provide this missing information, but difficulties in producing suitable samples from the hard, complex biocomposite have hindered progress. We have used focused ion beam (FIB) processing to produce precise cross sections from fully mineralized teeth (Fig. 1a) enabling us to apply a range of TEM techniques including imaging, selected area diffraction, energy-filtered TEM, electron energy-loss spectroscopy and high-angle annular dark field (HAADF) STEM imaging to obtain new insights into these biomaterials.

At the elemental level (Fig. 1b-d), energy-filtered TEM allows us to map compositional changes across the various interfaces and image the underlying organic matrix within the mineralized structure. At a structural level (Fig. 2), selected area diffraction has demonstrated that the biocomposite is more complex than previously reported, with an interleaved layer containing two iron oxyhydroxide phases (goethite and lepidocrocite) separating a soft apatite core from a hard outer magnetite layer, and an amorphous apatite layer at the iron oxyhydroxide interface [3].

This combination of FIB processing and TEM analysis provides significant structural and compositional information on these complex biocomposites at higher resolution than previously obtainable and will enhance future studies of these materials.

\section{References}

[1] J. A. Shaw et al., Microsc. Microanal. (in press) (2009).

[2] A.P. Lee, et al., J. Biol. Inorg. Chem. 3:614-619 (1998).

[3] M. Saunders, et al., J. Struct. Biol. (submitted) (2009).

[4] Work supported by Australian Research Council Discovery Grant \# DP0559858 D. J. Macey. 

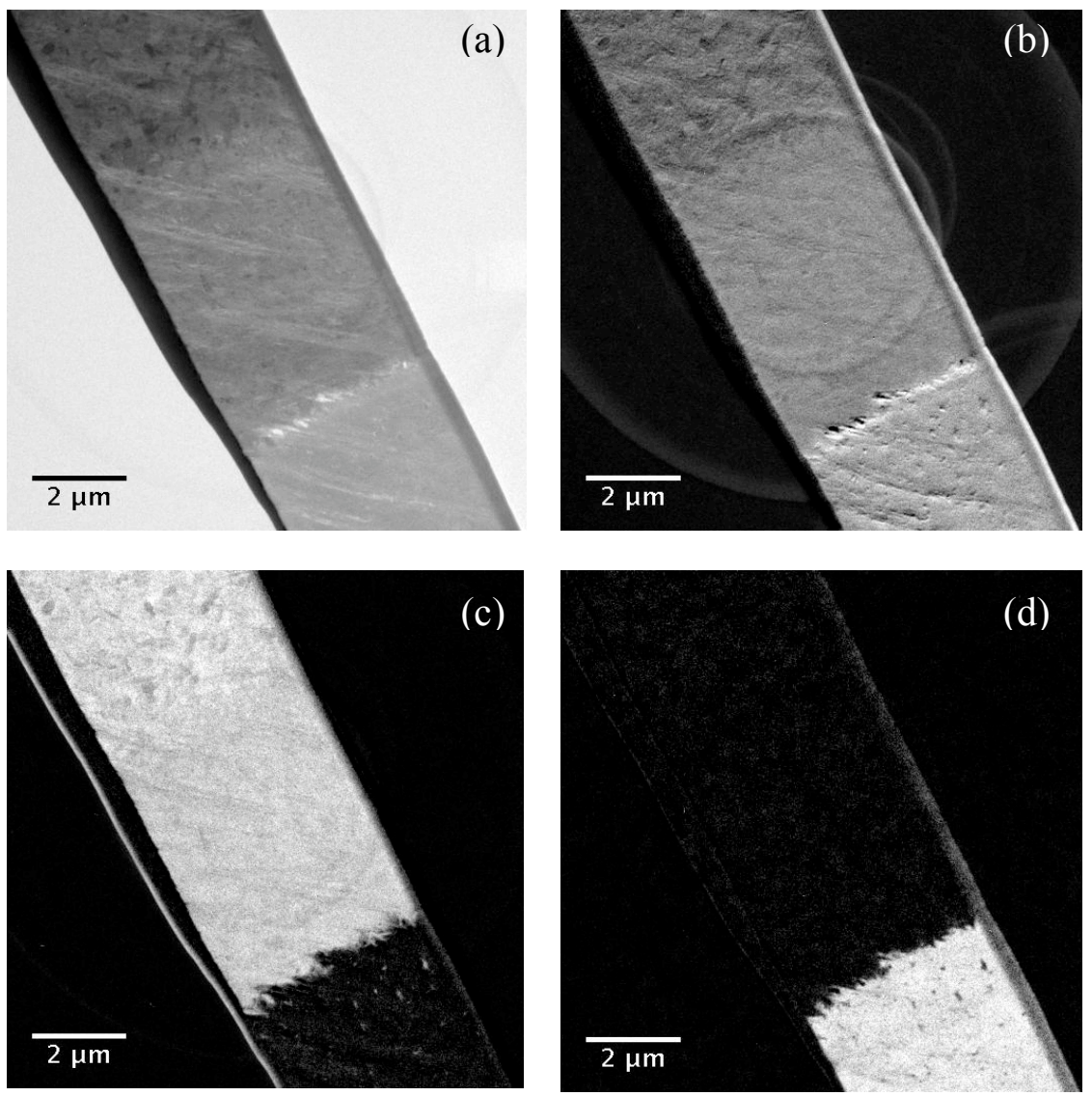

Fig. 1. (a) Bright field TEM image of FIB processed section of chiton tooth showing magnetite region (top left), iron oxyhydroxide region (middle layer) and apatite region (bottom right), with protective platinum and gold layers on surface (left edge); (b) Oxygen EFTEM elemental map obtained with O-K edge; (c) Iron EFTEM elemental map obtained with Fe- $\mathrm{L}_{2,3}$ edge; (d) Calcium EFTEM elemental map obtained with $\mathrm{Ca}-\mathrm{L}_{2,3}$ edge.
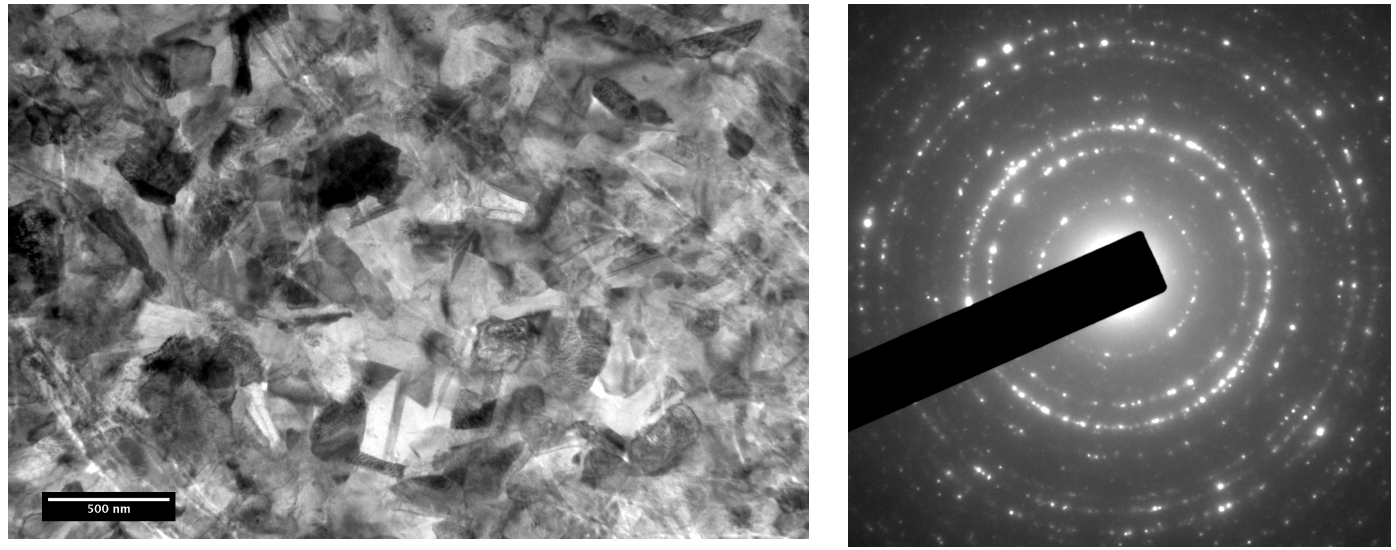

Fig. 2. Bright field image and selected area diffraction pattern from the magnetite layer confirming the polycrystalline nature of this region. Diffraction patterns from the other layers confirm the presence of goethite, lepidocrocite and apatite. 